(2) Open Access Full Text Article

\title{
Ozone oxidative postconditioning inhibits oxidative stress and apoptosis in renal ischemia and reperfusion injury through inhibition of MAPK signaling pathway
}

This article was published in the following Dove Press journal:

Drug Design, Development and Therapy

\section{Lei Wang \\ Zhiyuan Chen \\ Yang Liu \\ Yang Du \\ Xiuheng Liu}

Department of Urology, Renmin Hospital of Wuhan University,

Wuhan, Hubei, P. R. China
Correspondence: Zhiyuan Chen Department of Urology, Renmin Hospital of Wuhan University, No 238 Jiefang Road, Wuhan 430060, Hubei Province, P. R. China

Tel +86278 804 |9||

Fax +86 278804 I91।

Email chenzhiyuanI63@I63.com
Background: Ozone has been used as a curative agent for a variety of different diseases for over 150 years. In our previous study, we found that ozone oxidative preconditioning could alleviate renal damage induced by ischemia and reperfusion injury (I/R). Although this method had obvious protective effects in the reduction of $\mathrm{I} / \mathrm{R}$, its clinical application remains limited because this treatment must be commenced prior to the ischemic period, which is not practical in the clinic. Purpose: In the present study, we investigated whether ozone oxidative postconditioning (OzoneOP) could attenuate renal $\mathrm{I} / \mathrm{R}$ in vivo and in vitro, as well as the mechanisms underlying the effects of this treatment.

Methods: Sprague Dawley rats were subjected to right renal ischemia for $45 \mathrm{~min}$ and reperfusion for $24 \mathrm{~h}$, or to sham operation with the left kidney removed, both with and without OzoneOP. In addition, normal rat kidney tubular epithelial cells (NRK-52E) were chosen to create a hypoxia-reoxygenation $(\mathrm{H} / \mathrm{R})$ model of $3 \mathrm{~h}$ hypoxia and $24 \mathrm{~h}$ reoxygenation processes, both with or without OzoneOP and mitogen-activated protein kinase (MAPK) inhibitors.

Results: Our results showed that OzoneOP significantly reversed apoptosis and the abnormal superoxide dismutase and malondialdehyde levels induced by I/R or H/R. OzoneOP also inhibited activation of the MAPK pathways both in vivo and in vitro, which resulted in significant protection against apoptosis and oxidative stress.

Conclusion: Our current data provide evidence that OzoneOP might serve as a potential therapy for renal $\mathrm{I} / \mathrm{R}$.

Keywords: ozone, ischemia and reperfusion, MAPK

\section{Introduction}

Renal ischemia-reperfusion injury ( $\mathrm{I} / \mathrm{R})$ is a common cause of acute renal failure, which often arises from hypovolemic conditions, septic shock, accidental or iatrogenic trauma, cardiovascular surgery, and kidney surgery. ${ }^{1}$ The reperfusion process is vital in ischemic tissue. However, the process of ischemia causes damage to reperfusion. Numerous studies have suggested that oxidative stress and apoptosis play an important role in the pathogenesis of organic $\mathrm{I} / \mathrm{R}$ and result in cellular dysfunction. As the importance of oxidative stress and apoptosis in renal $\mathrm{I} / \mathrm{R}$ is becoming increasingly evident, it is essential to develop new therapies to prevent apoptosis and oxidative stress in cases of acute kidney injury.

Medical ozone has been shown to have curative effects in the treatment of a variety of different diseases over the last century. ${ }^{2}$ Indeed, numerous studies have indicated that 
ozone can convey different therapeutic effects. For example, ozone has anti-inflammatory properties and is known to act as a modulator of the antioxidant defense system and apoptosis. ${ }^{2-4}$ Ozone is also able to attenuate organic I/R and is a relatively simple and harmless treatment compared with other therapies. Recent studies, ${ }^{5,6}$ along with our own previous reports, ${ }^{7,8}$ have focused on the protective effects of ozone oxidative preconditioning against inflammation, apoptosis, and oxidative stress during $\mathrm{I} / \mathrm{R}$, both in vivo and in vitro. However, as it is unclear as to when tissue ischemia begins, the clinical application of preconditioning remains limited. As far as we know, the role of ozone oxidative postconditioning (OzoneOP) for renal I/R has yet to be reported.

The mitogen-activated protein kinase (MAPK) pathways, including extracellular signal-regulated kinase (ERK), c-Jun N-terminal kinase (JNK), and p38, play a key role in oxidative stress and apoptosis caused by I/R. ${ }^{9}$ A recent study found that the activation of p38 and ERK1/2 was involved in renal I/R injury. ${ }^{10}$ Furthermore, p38 MAPK was shown to serve as a nexus for signal transduction and, therefore, plays an important role in $\mathrm{I} / \mathrm{R}$ processes. Activation of $\mathrm{JNK} / \mathrm{p} 38$ is known to be critical in regulating the expression of cytokine and apoptotic protein through the activation of apoptosis signal-regulating kinase $1 .{ }^{11}$ In the present study, we investigated the role of OzoneOP on I/R-induced oxidative stress and apoptosis, both in vivo and in vitro. We also investigated the MAPK pathways related to these processes to determine whether and how OzoneOP provides protection against renal I/R injury.

\section{Materials and methods} Animal preparation

All adult Sprague Dawley rats (male, 220-250 g) were provided by the Center of Experimental Animals in the Medical College, Wuhan University. This project was approved by the committee of experimental animals of Wuhan University, and the procedures were carried out in accordance with routine animal-care guidelines. All procedures complied with the Guidelines for the Care and Use of Laboratory Animals. Before surgery procedures, rats were anesthetized with pentobarbital $(45 \mathrm{mg} / \mathrm{kg})$ and then placed on a homeothermic table to maintain core body temperature at $37^{\circ} \mathrm{C}$. All the rats then underwent a midline laparotomy followed by a right nephrectomy. Next, the left kidney was subjected to 45 min of ischemia with a nontrauma vascular clamp followed by $24 \mathrm{~h}$ of unclamping.

All animals were divided into three different groups: sham-operated (Sham) group, sham+O1 (Ozone $1 \mathrm{mg} / \mathrm{kg}$ ) group, sham+O2 (Ozone $2 \mathrm{mg} / \mathrm{kg}$ ) group, I/R group, I/R+O1 (Ozone $1 \mathrm{mg} / \mathrm{kg}$ ) group, and I/R+O2 (Ozone $2 \mathrm{mg} / \mathrm{kg}$ ) group.
Eight rats were allocated to each group. In the sham group, only the right kidneys were removed. In the sham+O1 group and the sham+O2 group, the right kidney was removed and the rats were administered with 15 ozone treatments by rectal insufflations ( 1 and $2 \mathrm{mg} / \mathrm{kg}$, respectively), once a day, as previously described. ${ }^{7}$ In the I/R group, the left kidney vessels were occluded using a clamp for $45 \mathrm{~min}$; reperfusion was then permitted for $24 \mathrm{~h}$ by unclamping. In the $\mathrm{I} / \mathrm{R}+\mathrm{O} 1$ and I/R+O2 groups, the vessels were unclamped and the rats administered with 15 ozone oxidative postconditioning treatments ( 1 and $2 \mathrm{mg} / \mathrm{kg}$, respectively), once a day. The concentration of ozone was $50 \mu \mathrm{g} / \mathrm{mL}$.

Then, the left kidneys were removed under fully maintained anesthesia and fixed in 10\% phosphate-buffered formalin or immediately stored at $-80^{\circ} \mathrm{C}$ to await subsequent analysis. Blood samples were also collected for the detection of blood urea nitrogen (BUN) and creatinine $(\mathrm{Cr})$ levels.

\section{Serum assays}

Assays were carried out using commercial kits and $2 \mathrm{~mL}$ of blood. All kits were used in accordance with the manufacturer's instructions (Nanjing Jiancheng Co., Nanjing, China). Levels of BUN and $\mathrm{Cr}$ were calculated using measurements acquired from a spectrophotometer.

\section{Superoxide dismutase (SOD) and malondialdehyde (MDA) measurement}

Commercial kits were used in accordance with the manufacturer's instructions (Nanjing Jiancheng Co., Nanjing, China) to measure SOD activity (xanthineoxidase method, catalog\# A001-3) and MDA concentration (thiobarbituric acid method, catalog\# A003-1).

\section{Histologic examinations}

Kidneys were first fixed and embedded, and then used to prepare $4-\mu m$-thick sections. Sections were then gradually deparaffinized and hydrated and stained with hematoxylin and eosin and terminal deoxynucleotidyl transferase-mediated deoxyuridine triphosphate-biotin nick end labeling (TUNEL) techniques. Morphologic assessments were observed by two experienced renal pathologists who were unaware of the treatments. An established grading scale of $0-4$, outlined by Jablonski et al, ${ }^{12}$ was used for the histopathologic assessment of I/R-induced damage.

\section{Immunohistochemistry}

The expression of bcl-2-associated $\mathrm{X}$ (BAX) and poly (ADP-ribose) polymerase 1 (PARP-1) was investigated by 
immunohistochemical staining. Sections were first deparaffinized and endogenous peroxidase activity was blocked with $3 \%$ hydrogen peroxide. Sections were then incubated with $10 \%$ normal goat serum in Tris-buffered saline (TBS) for $30 \mathrm{~min}$. Next, sections were incubated overnight with primary antibodies against BAX (1:100 dilution; Cell Signaling Technology, Boston, MA, USA) and PARP-1 (1:100 dilution; Abcam, Cambridge, MA, USA). The next day, sections were washed with PBS and then incubated with a horseradish peroxidase (HRP) 3,3'-diaminobenzidine (DAB) secondary antibody from the UltraVision Quanto Detection System (Thermo Fisher Scientific, Waltham, MA, USA) for $30 \mathrm{~min}$ at room temperature, followed by DAB. For quantitation, the relative mean integrated optical density (IOD) of each group was divided by the average IOD of the control, as determined by Image-Pro Plus software (version 6.0). All sections were photographed at a magnification of $\times 400$.

\section{Western blotting}

Proteins from renal tissue or cell cultures were extracted and quantified using the bicinchoninic acid method. Then, equivalent concentrations of protein ( $40 \mu \mathrm{g} /$ lane) were separated on $10 \%$ sodium dodecyl sulfate polyacrylamide gel electrophoresis gels and then transferred to a nitrocellulose membrane. Primary antibodies against p-ERK (sc-81492), ERK2 (sc-514302), p-JNK (sc-293136), JNK2 (sc-271133), c-fos (sc-52), p-p38 (sc-7973), and $\beta$-actin (sc-47778) were purchased from Santa Cruz (dilution 1:1,000), and p38 (\#8690), p-CREB (\#9198), and CREB (\#9197) were purchased from Cell Signaling Technology (dilution 1:1,000). $\beta$-actin was used as a loading control to ensure equal loading. Subsequently, membranes were washed twice with PBS and then incubated with goat anti-rabbit or goat anti-mouse HRPconjugated immunoglobulin G secondary antibody (1:2,000; ZDR-5306, ZDR-5307, ZSGB-BIO, Beijing, China) at room temperature for $1 \mathrm{~h}$. Specific bands were then visualized using Immobilon Western Chemiluminescence HRP substrate (Merck Millipore, Darmstadt, Germany). Optical densities were detected using Quantity One software (Bio-Rad, Hercules, CA, USA).

\section{Cell culture and the hypoxia- reoxygenation $(\mathrm{H} / \mathrm{R})$ model}

The renal tubular epithelial cell line, NRK-52E, was obtained from the Cell Bank of the Chinese Academy of Sciences (Shanghai, China). Prior to the H/R protocol, all cells in every group were cultured in $2 \mathrm{~mL}$ of serum-free medium for $24 \mathrm{~h}$ in order to be synchronized. The H/R injury protocol was then performed as previously described. ${ }^{13}$ Cells were then exposed to hypoxic $\left(\mathrm{O}_{2}\right.$ fraction below $\left.1 \%\right)$ condition for $3 \mathrm{~h}$ followed by normal conditions $\left(95 \%\right.$ air $\left./ 5 \% \mathrm{CO}_{2}\right)$ for $24 \mathrm{~h}$.

\section{Experimental groups of OzoneOP in vitro}

Ozone gas was delivered to biologic samples using a method described previously. ${ }^{14}$ The various concentrations of ozone gas were 20,30 , and $40 \mu \mathrm{g} / \mathrm{mL}$ of gas per $\mathrm{mL}$ of complete medium $(\mathrm{CM})$ with a predetermined volume. As ozone is very reactive, it is vital that the experimental steps must be precise and be carried out rapidly in order to obtain reproducible results. The samples were mixed with gas continuously for $20 \mathrm{~min}$.

All cells were divided into nine groups $(n=6)$. The control group and the $\mathrm{H} / \mathrm{R}$ group were washed with $\mathrm{PBS}$ and $1 \mathrm{~mL}$ of $\mathrm{CM}$ was added for $2 \mathrm{~h}$. The $\mathrm{H} / \mathrm{R}+\mathrm{O} 1$ group ( $20 \mu \mathrm{g} / \mathrm{mL}), \mathrm{H} / \mathrm{R}+\mathrm{O} 2$ group $(30 \mu \mathrm{g} / \mathrm{mL})$, and H/R+O3 group $(40 \mu \mathrm{g} / \mathrm{mL})$ were also washed; then $1 \mathrm{~mL}$ of $\mathrm{CM}$ was added for $2 \mathrm{~h}$. The H/R+SB-203580 group, H/R+SP-600125 group, and the $\mathrm{H} / \mathrm{R}+\mathrm{U}-0126$ group were first subjected to the $\mathrm{H} / \mathrm{R}$ process. Then, MAPK inhibitors, SB-203580 (Calbiochem), U-0126 (Calbiochem), and SP-600125 (Biomol), were added to each group in $1 \mathrm{~mL}$ of medium for $2 \mathrm{~h}$ at a concentration of $15 \mu \mathrm{M}$, or DMSO (D) as a control.

\section{Flow cytometry (FCM) assay}

FCM was used to analyze apoptosis in accordance with the manufacturer's instructions (KeyGEN, NanJing, China). After $24 \mathrm{~h}$ of reoxygenation, cells were collected and stained using fluorescein isothiocyanate-conjugated annexin $\mathrm{V}$ and propidium iodide for FCM detection.

\section{Immunocytochemistry staining}

After treatment, slides were washed twice with PBS and fixed in 4\% paraformaldehyde for $30 \mathrm{~min}$. After deparaffinization, endogenous peroxidase activity was blocked with $3 \%$ hydrogen peroxide. Slides were then incubated with $10 \%$ normal goat serum in TBS for $30 \mathrm{~min}$. The working concentration of BAX and PARP-1 was 1:100. After incubation with these antibodies at $4{ }^{\circ} \mathrm{C}$ overnight, the slides were washed three times with PBS and then incubated with secondary antibody, followed by the DAB color reagent.

\section{Statistical analysis}

Data are presented as mean \pm standard error of the mean. The means of the different groups were compared using one-way analysis of variance and the Student-Newman-Keuls test. 
Differences were considered to be statistically significant when $P<0.05$.

\section{Results}

\section{OzoneOP protected the kidney from I/R}

SD rats were subjected to sham operation, or $45 \mathrm{~min}$ of ischemia followed by $24 \mathrm{~h}$ reperfusion with or without OzoneOP. I/R induced renal dysfunction with a significant increase in the levels of $\mathrm{Cr}$ and BUN $(P<0.05)$. Furthermore, OzoneOP significantly prevented renal $\mathrm{I} / \mathrm{R}$, as demonstrated by an obvious decrease in the levels of $\mathrm{Cr}$ and BUN compared with the I/R group $(P<0.05$, Figure $1 \mathrm{~A}$ and $\mathrm{B})$, with more obvious effects observed at a concentration of $2 \mathrm{mg} / \mathrm{kg}$. No significant changes were observed between the sham, sham $+\mathrm{O} 1$, and sham $+\mathrm{O} 2$ groups. Renal I/R significantly increased MDA content and significantly decreased SOD activity $(P<0.05$, Figure $1 \mathrm{C}$ and D). Oxidative abnormalities were clearly ameliorated by treatment with OzoneOP $(P<0.05$, Figure $1 \mathrm{C}$ and $\mathrm{D})$. There were no significant differences in the levels of SOD or MDA when compared between the sham, sham $+\mathrm{O} 1$, and sham $+\mathrm{O} 2$ groups.

Renal tissue morphology is shown in Figure 1E and G. Renal tissues were normal in the sham, sham+O1, and sham $+\mathrm{O} 2$ groups. However, kidneys in the I/R group exhibited acute tubular damage in the proximal tubules, including tubular dilatation and loss of the brush border. OzoneOP protected the tubular epithelium from swelling and from loss of the brush border, with more obvious effects observed at a concentration of $2 \mathrm{mg} / \mathrm{kg}$.

\section{OzoneOP inhibited I/R-induced renal cell apoptosis}

Apoptotic cells in the kidneys were assayed by TUNEL staining (Figure 1F and H). There was a significantly higher

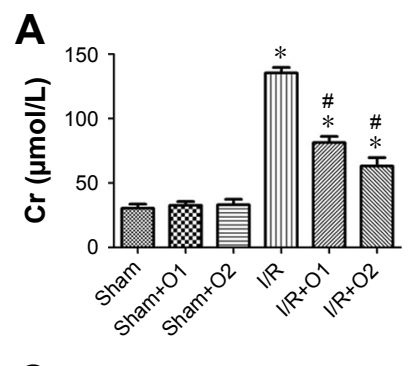

C
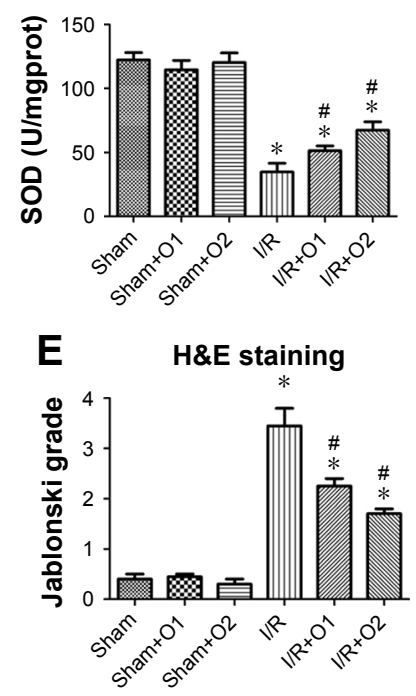

B

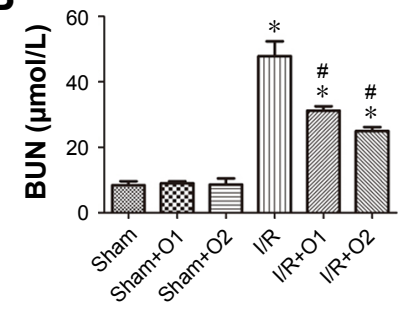

D

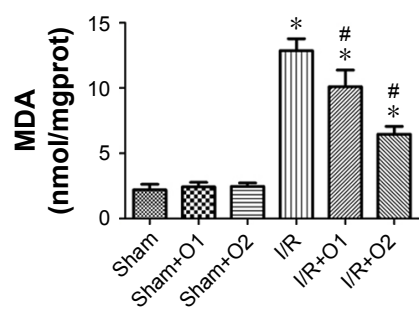

$\mathbf{F}$

TUNEL staining

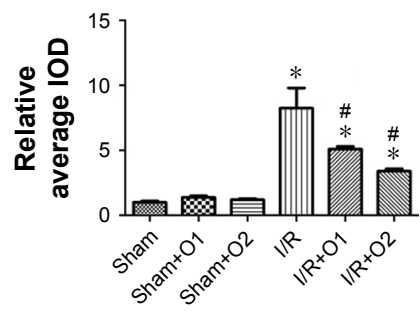

G

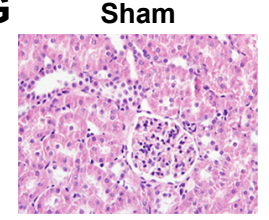

$\mathrm{I} / \mathrm{R}$

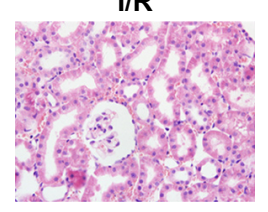

H

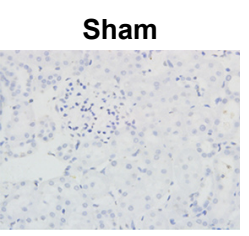

$\mathrm{I} / \mathrm{R}$

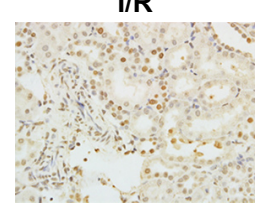

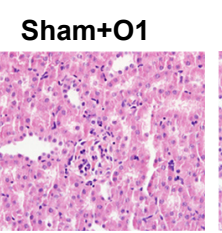

$\mathrm{I} / \mathrm{R}+01$

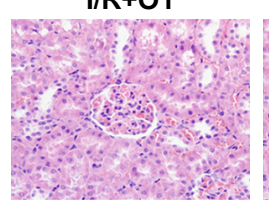

Sham+01

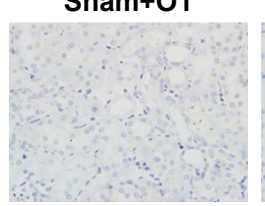

$\mathrm{I} / \mathrm{R}+\mathbf{0 1}$

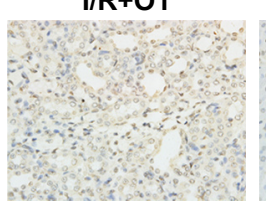

Sham+02

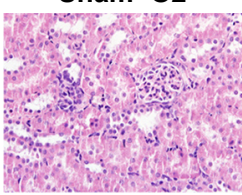

$\mathrm{I} / \mathrm{R}+\mathbf{0 2}$

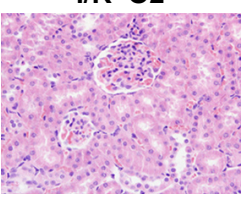

Sham+02

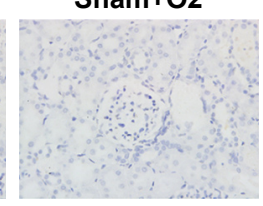

$\mathrm{I} / \mathrm{R}+\mathrm{O} 2$

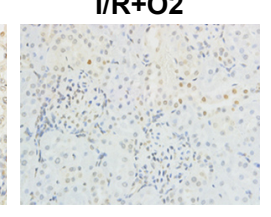

Figure I OzoneOP reduced the levels of urea and $\mathrm{Cr}$ and prevented renal oxidative stress apoptosis caused by I/R. Sprague Dawley rats were administered with vehicle or OzoneOP following surgery.

Notes: Serum and kidneys were subsequently collected for urea and Cr testing, H\&E staining, and TUNEL staining after $24 \mathrm{~h}$ of reperfusion. (A) Serum Cr concentration. (B) Serum BUN concentration. (C) SOD activity in renal tissue. (D) MDA concentration in renal tissue. (E) Jablonski grading scale scores after I/R. (F) Analyses of relative mean IOD using Image Pro-Plus software for TUNEL staining. (G) Representative images of H\&E staining of kidney sections (original magnification $\times 400$ ). (H) Representative images of TUNEL staining (brown nuclear staining) of kidney sections (original magnification $\times 400$ ). Data are presented as mean \pm standard error of the mean. $* P<0.05$ vs sham group; ${ }^{P} P<0.05$ vs I/R group.

Abbreviations: BUN, blood urea nitrogen; Cr, creatinine; H\&E, hematoxylin and eosin; IOD, integrated optical density; I/R, ischemia and reperfusion; MDA, malondialdehyde; OzoneOP, ozone oxidative postconditioning; SOD, superoxide dismutase; TUNEL, terminal deoxynucleotidyl transferase-mediated deoxyuridine triphosphate-biotin nick end labeling. 
number of TUNEL-positive cells in the I/R group than those receiving sham operations. However, significantly fewer positive cells were visible in the OzoneOP group $(P<0.05)$, which suggested that OzoneOP protected the kidney from apoptosis.
As shown in Figure 2A-D, after reperfusion for $24 \mathrm{~h}$, the expression of the proapoptotic proteins BAX and PARP-1 was increased. OzoneOP significantly reduced the expression of these proteins compared with those in the I/R group $(P<0.05)$.
A

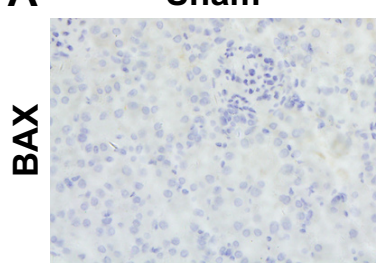

B

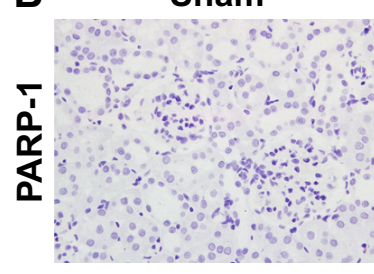

C

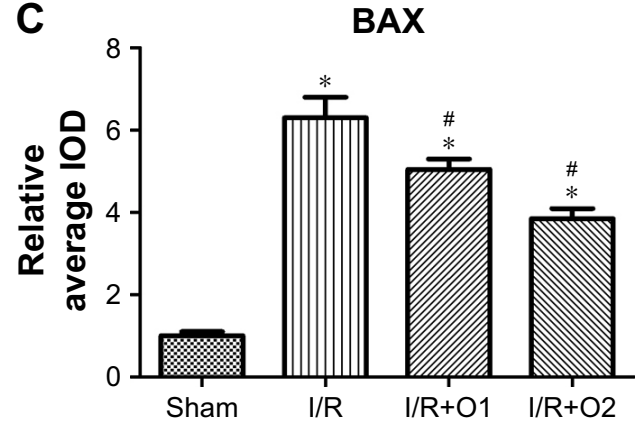

E

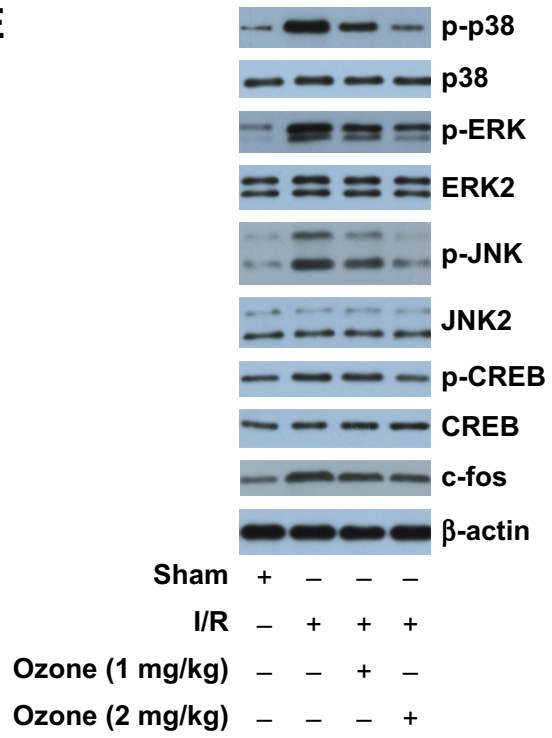

I/R

I/R

BAX
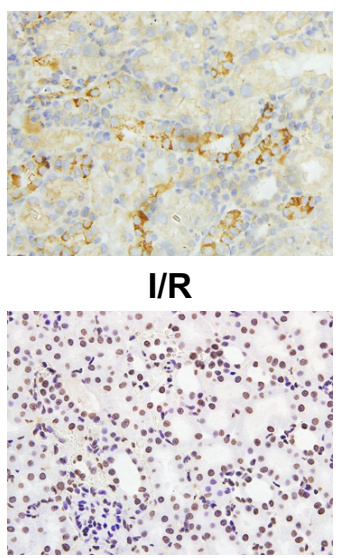

D

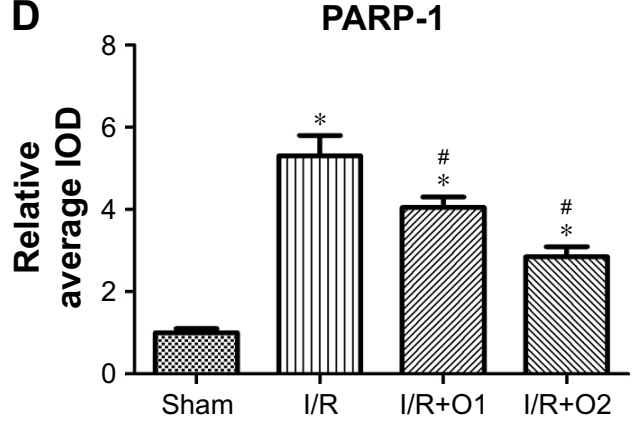

$F$

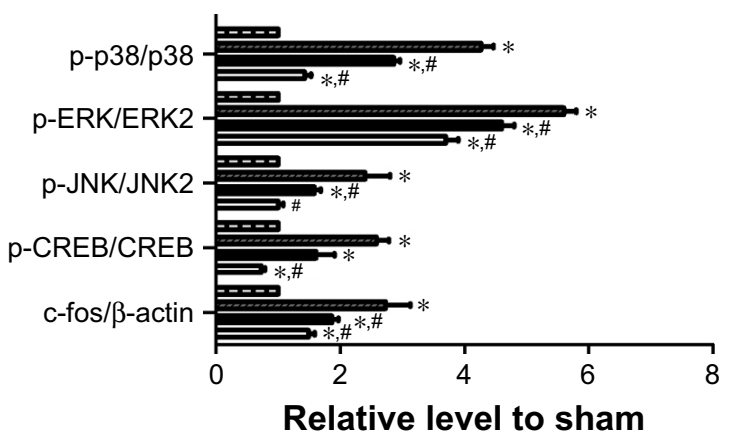

III Sham

DIA I/R

Ozone $(1 \mathrm{mg} / \mathrm{kg})$

Ozone (2 mg/kg)

Figure 2 OzoneOP reduced the expression of BAX and PARP-I and inhibited the MAPK signaling pathway in the kidney after I/R.

Notes: Sprague Dawley rats were administered with vehicle or OzoneOP after surgery. Kidneys were collected for immunohistochemistry and Western blotting after $24 \mathrm{~h}$ of reperfusion. (A, C) Representative images of BAX expression (brown cytoplasmic staining) in kidney sections (original magnification $\times 400$ ) and analyses of relative mean IOD. (B, D) Representative images of PARP-I expression (brown nuclear staining) in kidney sections (original magnification $\times 400$ ) and analyses of relative mean IOD. Representative blotting (E) and quantification of protein levels (F) are shown. Mean \pm standard error of the mean. ${ }^{*} P<0.05$ vs sham group; ${ }^{\#} P<0.05$ vs $\mathrm{I} / \mathrm{R}$ group.

Abbreviations: BAX, bcl-2-associated X; ERK, extracellular signal-regulated kinase; IOD, integrated optical density; I/R, ischemia and reperfusion; MAPK, mitogen-activated protein kinase; OzoneOP, ozone oxidative postconditioning; PARP-I, poly (ADP-ribose) polymerase I. 


\section{OzoneOP inhibited I/R-activated MAPK pathways}

The effects of I/R and OzoneOP on the phosphorylation of JNK, p38, ERK1/2, and CREB, and the expression of c-fos were investigated. As shown in Figure $2 \mathrm{E}$ and F, I/R promoted the phosphorylation of JNK, p38, ERK1/2, and CREB, and increased c-fos expression after reperfusion for $24 \mathrm{~h}$. OzoneOP significantly inhibited the I/R-induced activation of MAPK pathways, CREB and c-fos expression, with more obvious effects observed at a concentration of $2 \mathrm{mg} / \mathrm{kg}$.
OzoneOP protected NRK-52E cells against apoptosis and oxidative stress induced by $\mathrm{H} / \mathrm{R}$

Three hours of hypoxia followed by reoxygenation for $24 \mathrm{~h}$ resulted in obvious apoptosis compared with the control group (Figure $3 \mathrm{~A}$ and B). OzoneOP (20, 30, and $40 \mu \mathrm{g} / \mathrm{mL}$ ) exhibited a protective effect against apoptosis induced by H/R. Survival rate improved as the concentration of ozone increased, with more obvious effects observed at a concentration of $40 \mu \mathrm{g} / \mathrm{mL}$ (Figure 3A). Both the p38 inhibitor, SB-203580, and the JNK inhibitor, SP-600125, attenuated
A

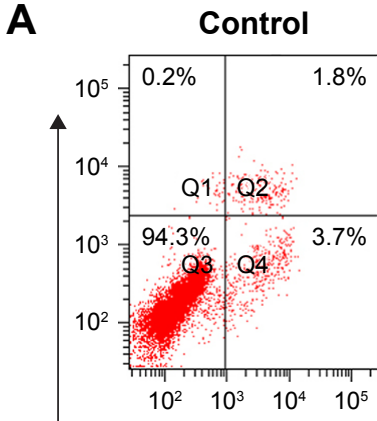

$\mathrm{H} / \mathrm{R}+01$

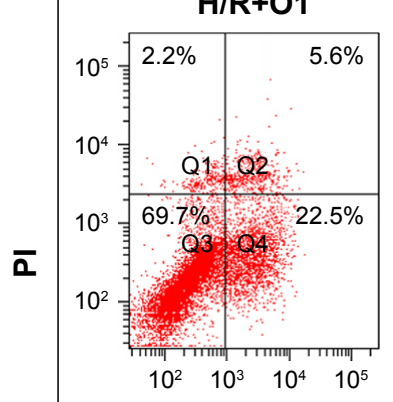

$\mathrm{H} / \mathrm{R}+\mathrm{SB}$

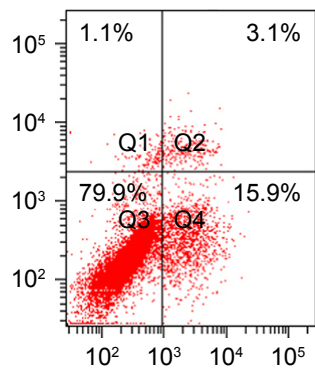

$H / R$

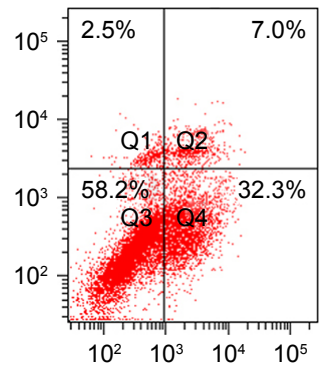

$\mathrm{H} / \mathrm{R}+\mathrm{O} 2$

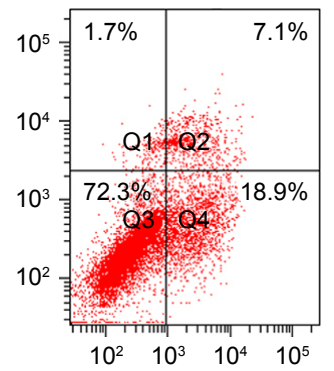

$\mathrm{H} / \mathrm{R}+\mathrm{SP}$

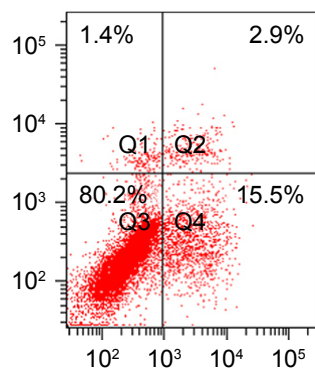

$H / R+D$

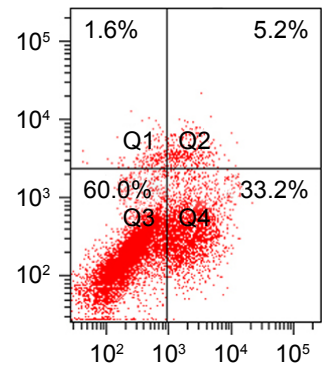

$\mathrm{H} / \mathrm{R}+\mathrm{O} 3$

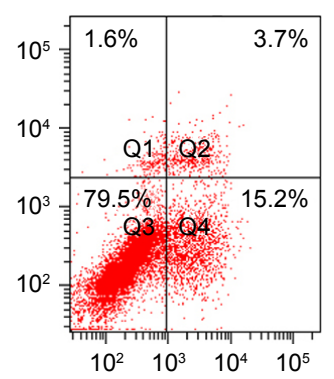

$\mathrm{H} / \mathrm{R}+\mathrm{U}$

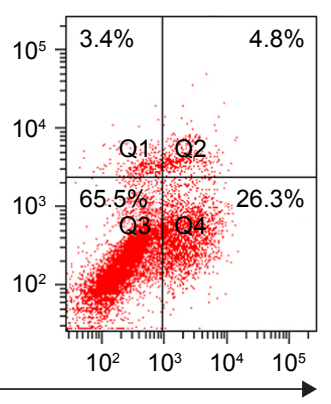

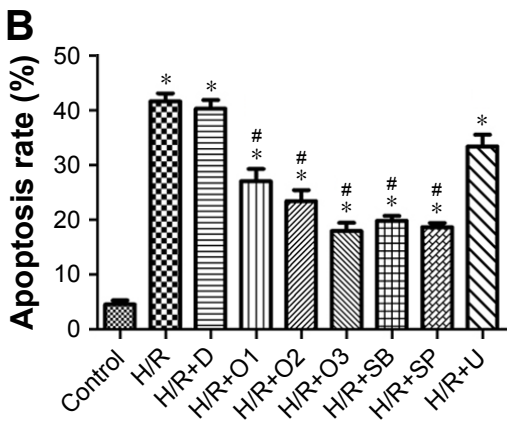

C

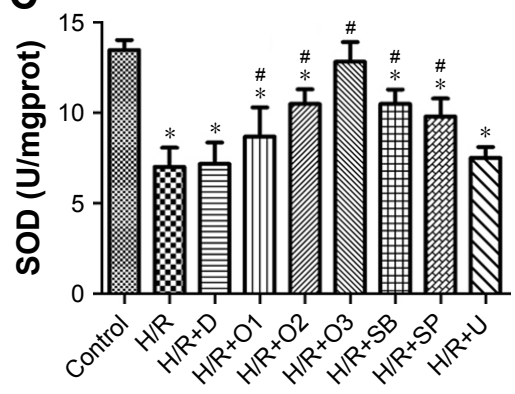

D

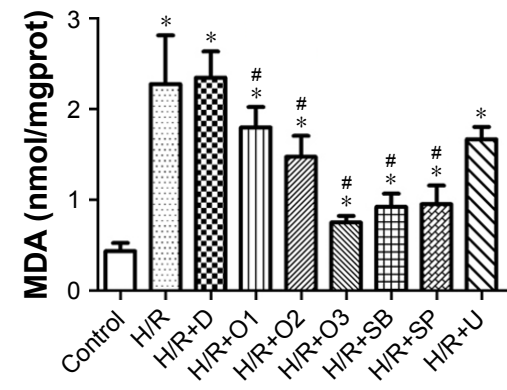

Annexin V FITC

Figure 3 OzoneOP protected NRK-52E cells against apoptosis and oxidative stress during hypoxia/reoxygenation.

Notes: NRK-52E cells were exposed to hypoxia for $3 \mathrm{~h}$ and then reoxygenation for $24 \mathrm{~h}$. Following reoxygenation, cells were maintained in complete medium for $2 \mathrm{~h}$ with 20, 30, and $40 \mu \mathrm{g} / \mathrm{mL}$ OzoneOP or the MAPK inhibitors, SB-203580 (SB), U-0I26 (U), and SP-600I25 (SP), at I5 $\mu$ M, or DMSO (D) as a control. Apoptosis was examined using annexin V-FITC/PI staining and flow cytometry analysis. Representative data (A) and quantification (B) are shown. (C) Assay of SOD activity in NRK-52E cells from different groups. (D) Assay of MDA content in NRK-52E cells from different groups. *P<0.05 vs control group; ${ }^{P}<<0.05$ vs $H / R$ group.

Abbreviations: DMSO, dimethyl sulfoxide; FITC, fluorescein isothiocyanate; MAPK, mitogen-activated protein kinase; MDA, malondialdehyde; NRK-52E, normal rat kidney tubular epithelial cells; OzoneOP, ozone oxidative postconditioning; PI, propidium iodide; SOD, superoxide dismutase; H/R, hypoxia-reoxygenation. 
cell apoptosis in NRK-52E cells exposed to H/R. However, the ERK inhibitor, U-0126, did not have a significant effect (Figure 3A).

As shown in Figure 3C and D, the MDA content in Group $\mathrm{H} / \mathrm{R}$ was significantly higher than those in the control group $(P<0.05)$, which was significantly attenuated by treatment with OzoneOP. SOD activity was significantly lower in Group H/R compared with the control group $(P<0.05)$, but OzoneOP inhibited the reduction in SOD levels induced by $\mathrm{H} / \mathrm{R}$ injury.

As shown in Figure 4A-D, the expression of the proapoptotic proteins BAX and PARP-1 were increased after $\mathrm{H} / \mathrm{R}$, although treatment with OzoneOP reduced their expression. SB-203580 and SP-600125 reversed the upregulated expression of BAX and PARP-1 in cells exposed to $\mathrm{H} / \mathrm{R}$, while U-0126 had no effect.

\section{OzoneOP inhibited H/R-activated MAPK pathways in NRK-52E cells}

The phosphorylation of p38, ERK1/2, and JNK was significantly increased in the $\mathrm{H} / \mathrm{R}$ group $(P<0.05)$. Treatment with OzoneOP significantly reduced the phosphorylation of these proteins in NRK-52E cells subjected to H/R in a dosedependent manner $(P<0.05$, Figure 4E-H).

\section{Discussion}

This study aimed to provide evidence that OzoneOP can alleviate renal $\mathrm{I} / \mathrm{R}$ injury and suggest indicators of the
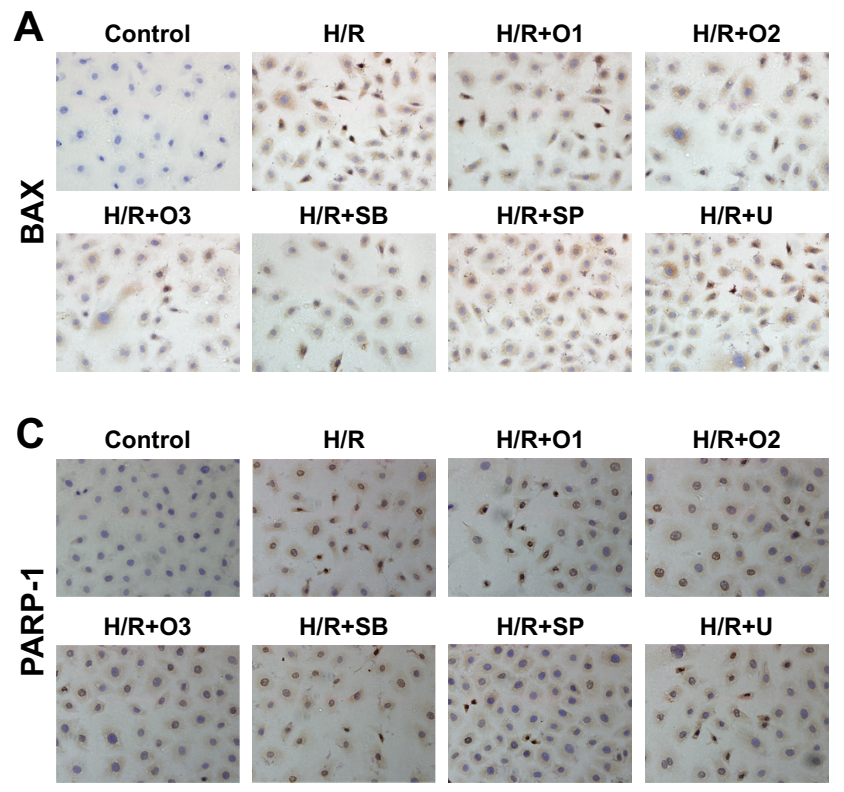

B

BAX

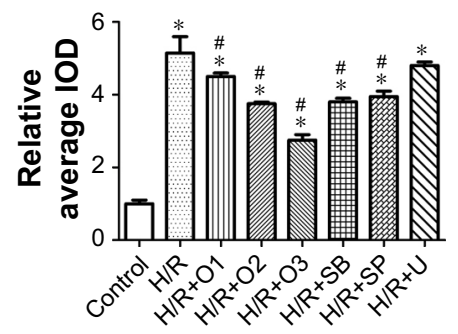

D

PARP-1

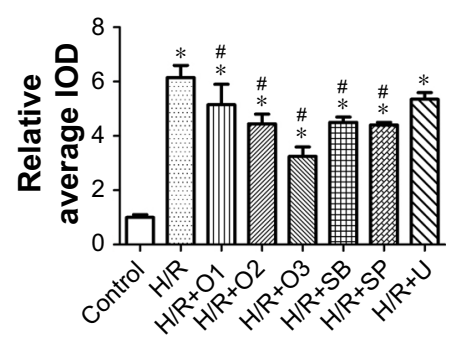

E

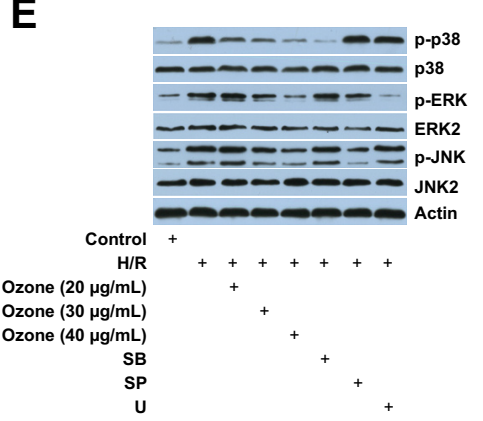

$F$

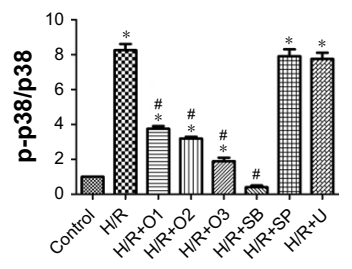

G

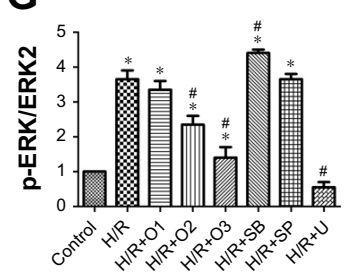

$\mathrm{H}$

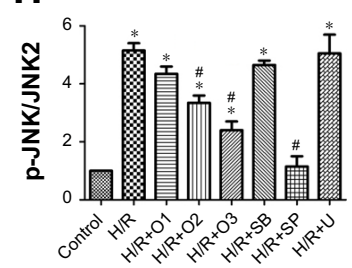

Figure 4 OzoneOP reduced the expression of BAX and PARP-I and inhibited the MAPK signaling pathways in NRK-52E cells exposed to H/R.

Notes: NRK-52E cells were administered with vehicle, and different concentrations of OzoneOP or the MAPK inhibitors, SB-203580 (SB), U-0I 26 (U), or SP-600I25 (SP), at a concentration of $15 \mu \mathrm{M}$ for $2 \mathrm{~h}$ after $\mathrm{H} / \mathrm{R}$ injury. The cells were collected for immunocytochemistry and Western blotting. (A, B) Representative images of BAX expression (brown cytoplasmic staining) of NRK-52E cells (original magnification $\times 400$ ) and analyses of relative mean IOD. (C, D) Representative images of PARP-I expression (brown nuclear staining) of NRK-52E cells (original magnification $\times 400$ ) and analyses of relative mean IOD. Representative blotting $(\mathbf{E})$ and quantification of protein levels $(\mathbf{F}-\mathbf{H})$ are shown. Mean \pm standard error of the mean. $* P<0.05$ vs control group; ${ }^{P}<0.05$ vs $H / R$ group.

Abbreviations: BAX, bcl-2-associated X; H/R, hypoxia/reoxygenation; IOD, integrated optical density; MAPK, mitogen-activated protein kinase; NRK-52E, normal rat kidney tubular epithelial cells; OzoneOP, ozone oxidative postconditioning; PARP-I, poly (ADP-ribose) polymerase I. 
possible mechanisms that mediate this effect. We evaluated the effect of OzoneOP using a classical animal model of I/R. Rat kidneys that had undergone I/R injury showed characteristic morphologic changes, including tubular dilatation and loss of the brush border. Furthermore, levels of urea and $\mathrm{Cr}$ in the $\mathrm{I} / \mathrm{R}$ group were clearly higher than in the control groups. The administration of OzoneOP improved these renal morphologic and functional changes, with more obvious effects observed at a concentration of $2 \mathrm{mg} / \mathrm{kg}$.

Oxidative stress plays an important role in mediating pathophysiologic responses in renal diseases and is characterized by increased production of reactive oxygen species (ROS) and reduced ROS scavenging capability. Normally, ROS generated from metabolic processes can be scavenged by endogenous antioxidant enzymes such as SOD,${ }^{15}$ which catalyze the dismutation of the superoxide radical to hydrogen peroxide. In addition, MDA indicates the extent of cell injury caused by oxidative stress. Following I/R injury, the overproduction of ROS can result in cellular dysfunction, including the oxidation of lipids, proteins, and nucleic acids. ${ }^{16}$ In our present study, we determined the levels of oxidative stress in an animal model of $\mathrm{I} / \mathrm{R}$ by measuring SOD activity and MDA concentration. While MDA levels were significantly higher in renal tissue subjected to I/R, OzoneOP treatment led to a significant reduction in these levels. Furthermore, SOD activity was significantly decreased in the I/R group; this effect was reversed by OzoneOP treatment, which indicated that OzoneOP may normalize the redox status of renal cells under $\mathrm{I} / \mathrm{R}$.

In vitro $\mathrm{H} / \mathrm{R}$ represents a laboratory version of animal $\mathrm{I} / \mathrm{R}$ models. ${ }^{17}$ To confirm the protective effects of OzoneOP on $\mathrm{I} / \mathrm{R}$ which we observed in our in vivo model, we chose NRK-52E cells to undergo $\mathrm{H} / \mathrm{R}$ injury; we based this decision on our previous research. ${ }^{8}$ NRK-52E cells are susceptible to H/R, which originates from the renal tubular epithelium in experimental rats. Moreover, proximal epithelium damage is an important reason for renal dysfunction. In our in vitro model, we found that cells in the H/R group showed increased MDA production and reduced SOD activity. However, these changes could be reversed by OzoneOP treatment. These findings suggest that the overproduction of ROS plays a central role in kidney cell injury induced by $\mathrm{H} / \mathrm{R}$ and that OzoneOP may offer some level of protection through antioxidant properties.

Apoptosis, a form of cell death, has been demonstrated to occur after renal $\mathrm{I} / \mathrm{R}^{18}$ and is characterized by the activation of either extrinsic or intrinsic pathways. BAX, as an important member of the family of Bcl-2 proteins, can promote apoptosis by forming oligomers on the outer mitochondrial membrane and forming a channel for the release of cyto-c. ${ }^{19}$ PARP-1 also plays a critical role during apoptosis after $\mathrm{I} / \mathrm{R}$ injury $;^{20}$ the $116 \mathrm{kDa}$ PARP-1 protein is cleaved into an $89 \mathrm{kDa}$ apoptotic fragment during apoptosis. Increased levels of ROS have also been demonstrated to induce depolarization of the mitochondrial membrane, which eventually generates proapoptotic molecules in cells. ${ }^{21}$ Our previous studies have demonstrated that ozone oxidative preconditioning exerts protective effects in a rat model of I/R injury, specifically by inhibiting inflammation and apoptosis. ${ }^{7}$ In the present study, our TUNEL staining data indicated that $\mathrm{I} / \mathrm{R}$ injury obviously induced apoptosis when compared with the sham-operated group. The expression of the apoptotic proteins, BAX and PARP-1, was significantly increased in the I/R group; however, OzoneOP could reduce these increased levels of expression. Moreover, our in vitro study indicated that the rate of apoptosis was obviously higher in the H/R group compared with the control and DMSO groups. Furthermore, the expression of BAX and PARP-1 in the H/R group was higher than those in the control group. However, OzoneOP could reverse these changes induced by $\mathrm{H} / \mathrm{R}$. These findings suggest that OzoneOP can protect kidney cells against apoptosis caused by $\mathrm{I} / \mathrm{R}$ both in vivo and in vitro.

The activation of MAPKs will activate numerous transcription factors, which are critical molecular targets for pharmacologic intervention. For example, ERK1/2 is known to be related to cell proliferation and growth. ${ }^{22}$ One substrate for ERK $1 / 2$ is the transcription factor CREB, which is associated with cell survival. ${ }^{23}$ Furthermore, JNK and p38 are generally activated through environmental changes, including oxidative stress and inflammatory cytokines. ${ }^{22}$ In our study, reperfusion of the rat kidney for $24 \mathrm{~h}$, or the exposure of kidney cells to reoxygenation for $24 \mathrm{~h}$, resulted in a significant increase in the phosphorylation of JNK, p38, and ERK1/2. Postconditioning with ozone reduced the phosphorylation of all three molecules. While SB-203580 and SP-600125 inhibited cell apoptosis induced by H/R, U-0126 had no specific effect. These lines of evidence showed that OzoneOP may inhibit cell apoptosis through multiple mechanisms, but predominantly involving p38 and JNK. In an earlier paper it was reported that the nuclear factor kB (NF-kB) inhibitor, Bay 11-8082, reduced levels of phosphorylation for the ERK1/2, p38, and JNK. In future works, we will further verify the relationship between NF-kB and the MAPK pathway in renal I/R.

\section{Conclusion}

In conclusion, the present study provides, for the first time, evidence that ozone oxidative postconditioning exerts a 
significant renal protective effect against I/R injury by attenuating oxidant stress and apoptosis in kidney cells.

\section{Acknowledgments}

This study was supported by Wuhan Morning Light Plan of Youth Science and Technology (2017050304010281), Hubei Province Health and Family Planning Scientific Research Project (No WJ2017M025 and No WJ2017Z005), Natural Science Foundation of Hubei Province (2017CFB181), and Research Project of Wuhan University (No 2042017kf0097).

\section{Author contributions}

Lei Wang performed the experiments and analyzed the data; Zhiyuan Chen conceived and designed the experiments; Yang Du and Yang Liu analyzed the data and Lei Wang contributed to the writing of the manuscript. All authors contributed toward data analysis, drafting and critically revising the paper and agree to be accountable for all aspects of the work. All the authors read and approved the final version of the manuscript.

\section{Disclosure}

The authors report no conflicts of interest in this work.

\section{References}

1. Wang L, Chen H, Liu XH, et al. Ozone oxidative preconditioning inhibits renal fibrosis induced by ischemia and reperfusion injury in rats. Exp Ther Med. 2014;8(6):1764-1768.

2. Elvis AM, Ekta JS. Ozone therapy: a clinical review. J Nat Sci Biol Med. 2011;2(1):66-70

3. Wu X, Li Z, Liu X, et al. Major ozonated autohemotherapy promotes the recovery of upper limb motor function in patients with acute cerebral infarction. Neural Regen Res. 2013;8(5):461-468.

4. Caliskan B, Guven A, Ozler M, et al. Ozone therapy prevents renal inflammation and fibrosis in a rat model of acute pyelonephritis. Scand J Clin Lab Invest. 2011;71(6):473-480.

5. Ajamieh HH, Menendez S, Martinez-Sanchez G, et al. Effects of ozone oxidative preconditioning on nitric oxide generation and cellular redox balance in a rat model of hepatic ischaemia-reperfusion. Liver Int. 2004;24(1):55-62.

6. Ajamieh HH, Berlanga J, Merino N, et al. Role of protein synthesis in the protection conferred by ozone-oxidative-preconditioning in hepatic ischaemia/reperfusion. Transpl Int. 2005;18(5):604-612.
7. Chen H, Xing B, Liu X, et al. Ozone oxidative preconditioning inhibits inflammation and apoptosis in a rat model of renal ischemia/reperfusion injury. Eur J Pharmacol. 2008;581(3):306-314.

8. Wang L, Chen H, Liu XH, et al. The protective effect of ozone oxidative preconditioning against hypoxia/reoxygenation injury in rat kidney cells. Ren Fail. 2014;36(9):1449-1454.

9. Chen J, Wang W, Zhang Q, et al. Low molecular weight fucoidan against renal ischemia-reperfusion injury via inhibition of the MAPK signaling pathway. PLoS One. 2013;8(2):e56224.

10. Zhang J, Xia J, Zhang Y, et al. HMGB1-TLR4 signaling participates in renal ischemia reperfusion injury and could be attenuated by dexamethasone-mediated inhibition of the ERK/NF-kappaB pathway. Am J Transl Res. 2016;8(10):4054-4067.

11. Yuan ZQ, Feldman RI, Sussman GE, et al. AKT2 inhibition of cisplatin-induced JNK/p38 and Bax activation by phosphorylation of ASK1: implication of AKT2 in chemoresistance. J Biol Chem. 2003; 278(26):23432-23440.

12. Jablonski P, Howden BO, Rae DA, et al. An experimental model for assessment of renal recovery from warm ischemia. Transplantation. 1983;35(3):198-204.

13. Sauvant C, Schneider R, Holzinger H, Renker S, Wanner C, Gekle M. Implementation of an in vitro model system for investigation of reperfusion damage after renal ischemia. Cell Physiol Biochem. 2009; 24(5-6):567-576.

14. Valacchi G, Bocci V. Studies on the biological effects of ozone: 11 Release of factors from human endothelial cells. Mediators Inflamm. 2000; 9(6):271-276.

15. Meng FJ, Hou ZW, Li Y, Yang Y, Yu B. The protective effect of picroside II against hypoxia/reoxygenation injury in neonatal rat cardiomyocytes. Pharm Biol. 2012;50(10):1226-1232.

16. Ferrari R, Ceconi C, Curello S, Cargnoni A, Pasini E, Visioli O. The occurrence of oxidative stress during reperfusion in experimental animals and men. Cardiovasc Drugs Ther. 1991;5(Suppl 2):277-287.

17. Zager RA, Johnson AC, Hanson SY, Lund S. Ischemic proximal tubular injury primes mice to endotoxin-induced TNF-alpha generation and systemic release. Am J Physiol Renal Physiol. 2005;289(2):F289-F297.

18. Wolfs TG, de Vries B, Walter SJ, et al. Apoptotic cell death is initiated during normothermic ischemia in human kidneys. Am J Transplant. 2005;5(1):68-75.

19. Havasi A, Borkan SC. Apoptosis and acute kidney injury. Kidney Int 2011;80(1):29-40.

20. Song ZF, Ji XP, Li XX, Wang SJ, Wang SH, Zhang Y. Inhibition of the activity of poly (ADP-ribose) polymerase reduces heart ischaemia/ reperfusion injury via suppressing JNK-mediated AIF translocation. J Cell Mol Med. 2008;12(4):1220-1228.

21. Circu ML, Aw TY. Reactive oxygen species, cellular redox systems, and apoptosis. Free Radic Biol Med. 2010;48(6):749-762.

22. Johnson GL, Lapadat R. Mitogen-activated protein kinase pathways mediated by ERK, JNK, and p38 protein kinases. Science. 2002;298(5600):1911-1912

23. Bonni A, Brunet A, West AE, et al. Cell survival promoted by the RasMAPK signaling pathway by transcription-dependent and -independent mechanisms. Science. 1999;286(5443):1358-1362.

\section{Publish your work in this journal}

Drug Design, Development and Therapy is an international, peerreviewed open-access journal that spans the spectrum of drug design and development through to clinical applications. Clinical outcomes, patient safety, and programs for the development and effective, safe, and sustained use of medicines are the features of the journal, which

\section{Dovepress}

has also been accepted for indexing on PubMed Central. The manuscript management system is completely online and includes a very quick and fair peer-review system, which is all easy to use. Visit http://www.dovepress.com/testimonials.php to read real quotes from published authors. 